\title{
Effect of heat treatment on small scale fragmentation of aluminium alloy
}

\section{R. Edwards* and C. Deal}

Small scale explosions, using a detonator, of 7075 aluminium alloy cylinders, $15-100 \mathrm{~mm}$ outside diameter, were carried out to investigate the effects of heat treatment on fragmentation. This was the finest for the strongest as received alloy and coarsest for the softest overaged alloy. This effect was similar to that seen in investigations of the fragmentation of steel. Cylinders of 50 and $100 \mathrm{~mm}$ in diameter did not fragment but plastically deformed with maximum deformation at the cylinder bottom. Fragmentation of 33 and $42 \mathrm{~mm}$ diameter cylinders produced long fragments typical of the break-up of thick walled cylinders. At smaller diameters, break-up gave fragments of several shapes, finer fragments being largely associated with the smallest diameter cylinders and the highest strength alloys. Results followed those seen in large scale studies of cylinder break-up and suggest the possibility of using small scale fragmentation experiments in the investigation of the effects of composition, heat treatment and processing on natural fragmentation.

Keywords: Fragmentation, Aluminium alloy, Heat treatment

\section{List of symbols}

$\begin{aligned} c & \text { acoustic velocity } \\ K_{\mathrm{f}} & \text { fragmentation toughness } \\ P_{2} & \text { constant in stress-strain curve } P=P_{1}+ \\ & P_{2} \ln (1+\varepsilon) \\ r & \text { radius of ring bomb at break-up } \\ S & \text { fragment characteristic length } \\ V & \text { velocity of ring bomb at break-up } \\ x_{\text {mean }} & \text { mean length of fragment } \\ \dot{\varepsilon} & \text { strain rate } \\ \varepsilon_{\mathrm{F}} & \text { true fracture strain } \\ \rho & \text { density } \\ \sigma_{\mathrm{F}} & \text { true fracture stress }\end{aligned}$

\section{Introduction}

Fragmentation has been most often studied in the context of bombs or shells where the mass of explosive charge: mass of metal ratio $(C / M)$ is large in order to maximise the initial fragment velocity. ${ }^{1}$ It will be of interest to study the fragmentation process for a wider range of values of $C / M$ to investigate how this is affected by both the $C / M$ ratio and the mechanical properties of the alloy. Recent work $^{2-4}$ has emphasised that material behaviour at high rates of strain involves phenomena, such as dynamic twinning, ductile to brittle transformations and spalling, which make the modelling of phenomena more difficult with measurements and

Department of Applied Science, Security and Resilience, Cranfield University, Defence Academy of the United Kingdom, Shrivenham, Swindon SN6 8LA, UK

*Corresponding author, email m.r.edwards@cranfield.ac.uk calculations becoming more difficult as the strain rate increases.

Mott ${ }^{5}$ studied the fragmentation of thin walled ring bombs, thus being able to characterise the fragments in terms of their average length. He found that the average length was given by

$$
x_{\text {mean }}=\alpha \sigma_{\mathrm{F}}\left(\frac{1+\varepsilon_{\mathrm{F}}}{\rho} P_{2}\right)^{1 / 2} \frac{r}{V}
$$

This shows that small fragments were associated with low true strains to fracture and high workhardening rates, properties which can be modified by changes of alloy composition or heat treatment. Additionally a high dial velocity (fragment velocity) at the point of fracture, controlled, as determined by Gurney, ${ }^{1}$ by the nature of the explosive and the $C / M$ ratio, will produce small fragments. Thus, shells that are designed to produce smaller fragments will tend to be manufactured from steels of higher carbon content than shells that produce larger fragments.

Another approach, due to Grady and Olson, ${ }^{6,7}$ considered an energy balance during fragmentation and set the fragment characteristic length as

$$
S=\left[\frac{\left(24 K_{\mathrm{f}}\right)^{1 / 2}}{\rho c \dot{\varepsilon}}\right]^{2 / 3}
$$

Here small fragments are associated with low values of fragmentation toughness, which, as a first approximation, can be regarded as associated with materials of low fracture toughness. This is often consistent with the predictions of Mott, since materials with low fracture strains are likely to have low fracture toughnesses.

In natural fragmentation, there is a wide range of fragment sizes. Mock and Holt ${ }^{8}$ showed that there was a

(C) 2009 Institute of Materials, Minerals and Mining

Published by Maney on behalf of the Institute

Received 9 July 2009; accepted 30 July 2009

DOI 10.1179/026708309X12506933873341 
taxonomy of fragment types depending on whether the outer and/or inner cylinder surfaces were present or absent in the fragment. The largest fragments tended to have both outer and inner surfaces present, while the smallest would have neither cylinder surface present. Thus, the distribution of fragment sizes would be a composite of up to four separate distributions, corresponding to the four types of fragment. This concept of fragment type helps to explain the Grady and Kipp's 9,10 concept of statistically inhomogeneous fragmentation.

Studies $^{11}$ of the fracturing of thick walled annealed AISI 1020 steel cylinders by the RDX based explosive C3 show that fracture did not occur at $C / M$ of 0.0025 , while for $0 \cdot 067 \geqslant C / M \geqslant 0 \cdot 006$, fracture occurred. In all cases, the fragments were long, extending over the length of the cylinder. For the case where fracture did not occur, there was evidence of plastic deformation, shown by enlargement of the central hole. Additionally, fractures were present within the walls of the tube, but these did not connect with the outer or inner surfaces of the tube. The number of fractures was estimated ${ }^{12}$ to be proportional to the particle velocity and inversely proportional to the material critical impact velocity. This implies that increasing the strength of an alloy by heat treatment will reduce the critical impact velocity and therefore increase the number of fractures. Additionally, increasing the $C / M$ ratio by decreasing the external diameter should increase the particle velocity and therefore increase the number of fractures.

Fragmentation trials tend to be expensive when carried out at full scale. It is of interest to investigate the process where the amount of explosive is very small since it may be then be possible to carry out the trial in a simple container. Work carried out using the cartridge test $^{13-15}$ has aimed to use a measure of the damage created in a cartridge case as a method of determining the explosive power of compounds, principally those seen in improvised explosives.

Walsh ${ }^{16}$ showed in a trial of cylinders packed with explosive that both the number of fragments and their average mass increased at constant $C / M$ ratio as the wall thickness increased. This effect was more marked for tool steel than for an annealed medium carbon steel and appeared to be associated with a decreased proportion of fragments containing outside and inside surfaces of the shell (type 1 according to the taxonomy of Mock and Holt). He also showed that for a constant wall thickness, the increase in the average fragment mass with increasing $C / M$ ratio is relatively small. All of these experiments were carried out with $C / M$ ratios of $0 \cdot 14-0 \cdot 56$. Since steel is the customary material for shells and bombs, most studies of heat treatment effects on fragmentation have been carried out on steel samples. However, safety analyses of aluminium alloy components containing energetic materials will involve the evaluation of likely fragmentation of the aluminium alloy. The aim of the present work is to look at the fragmentation behaviour

Table 1 Hardness test results

Hardness, HV0.3

\begin{tabular}{llll}
\cline { 2 - 4 } $\begin{array}{l}\text { Cylinder diameter, } \\
\mathbf{m m}\end{array}$ & As received & $\begin{array}{l}\text { Solution } \\
\text { treated }\end{array}$ & Overaged \\
\hline 25 & $202 \pm 12$ & $160 \pm 5$ & $109 \pm 6$ \\
100 & $191 \pm 9$ & $142 \pm 7$ & $107 \pm 6$ \\
\hline
\end{tabular}

Table 2 Tensile test results (25 $\mathrm{mm}$ diameter cylinders)

\begin{tabular}{lccc}
\hline & 25AR & 25ST & 250A \\
\hline Tensile strength, MPa & $578 \pm 12$ & $551 \pm 22$ & $343 \pm 1.4$ \\
Yield strength, MPa & $519 \pm 13$ & $401 \pm 15$ & $274 \pm 41$ \\
True fracture strength, MPa & $623 \pm 27$ & $610 \pm 45$ & $356 \pm 13$ \\
Elongation, \% & $16 \pm 0.9$ & $16 \pm 1.4$ & $23 \pm 0.9$ \\
Reduction in area, \% & $8 \pm 2.4$ & $10 \pm 1.8$ & $30 \pm 0.0$ \\
True fracture strain & $0.08 \pm 0.03$ & $0.11 \pm 0.02$ & $0.36 \pm 0.0$ \\
\hline
\end{tabular}

as a function of the $C / M$ ratio and how this is affected by the heat treatment of the aluminium alloy.

\section{Materials and methods}

\section{Materials used}

The alloy was the $\mathrm{Al}-\mathrm{Zn}-\mathrm{Mg}-\mathrm{Cu}$ alloy 7075 with a nominal composition of $\mathrm{Al}-5 \cdot 6 \mathrm{Zn}-2 \cdot 5 \mathrm{Mg}-1 \cdot 6 \mathrm{Cu}$.

The heat treatments were:

(i) AR: as received, solution treated and peak aged (T6 temper)

(ii) ST: solution treated for $2 \mathrm{~h}$ at $480^{\circ} \mathrm{C}$, water quenched

(iii) $\mathrm{OA}$ : overaged, solution treated for $2 \mathrm{~h}$ at $480^{\circ} \mathrm{C}$, water quenched and aged for $48 \mathrm{~h}$ at $200^{\circ} \mathrm{C}$.

Hardness testing was carried out using an Indentec hardness testing machine HWDM-7 with a $300 \mathrm{~g}$ load. Ten measurements were made on each sample and results are shown in Table 1. The measurements were taken from the outside edge to inside hole at the ends of the samples.

Tensile testing was carried out on samples with a diameter of $4.53 \mathrm{~mm}$ and a gauge length of $16 \mathrm{~mm}$. Crosshead speed was $2 \mathrm{~mm} \mathrm{~min}^{-1}$, corresponding to a nominal strain rate of $2 \times 10^{-3} \mathrm{~s}^{-1}$. Average results for samples taken from the $25 \mathrm{~mm}$ diameter bars are in Table 2 and for samples taken from the $100 \mathrm{~mm}$ bar in Table 3. In all cases, three specimens were taken for each heat treatment condition.

\section{Experimental methods}

The specimens were in the form of cylinders of 15-100 mm outside diameter with a $6.5 \mathrm{~mm}$ diameter hole in the centre. The length of the cylinders was $35 \mathrm{~mm}$, which corresponded to the length of the explosive in the detonators used. The $C / M$ ratios for the experiments are listed in Table 4, assuming a charge mass of $1.05 \mathrm{~g}$ and ignoring the mass of the thin aluminium tube of the detonator.

The explosive charge was a single electrical L2A1 detonator which consists of a tube, a main filling, a primary charge and a fuzehead assembly. A diagram of the detonator can be seen at Fig. 1 .

The tube is manufactured from aluminium, $61 \mathrm{~mm}$ long and $6.5 \mathrm{~mm}$ in diameter. It is closed at one end and contains the main filling, the primary charge and the fuzehead assembly. The main filling is made up of $1.05 \mathrm{~g}$ CE (tetryl) which is in the form of one granulated pellet with a nominal mass of $0.3 \mathrm{~g}$ and three pressed pellets

Table 3 Tensile test results $(100 \mathrm{~mm}$ diameter cylinders)

\begin{tabular}{lccr}
\hline & 100AR & 100ST & 1000A \\
\hline Tensile strength, MPa & $592 \pm 43$ & $582 \pm 7$ & $368 \pm 2$ \\
Yield strength, MPa & $524 \pm 42$ & $399 \pm 6$ & $268 \pm 1$ \\
True fracture strength, MPa & $626 \pm 94$ & $661 \pm 10$ & $302 \pm 26$ \\
Elongation, \% & $19 \pm 1.0$ & $21 \pm 1.7$ & $25 \pm 0.0$ \\
Reduction in area, \% & $10 \pm 1.5$ & $11 \pm 1.7$ & $30 \pm 0.0$ \\
True fracture strain & $0.11 \pm 0.01$ & $0.12 \pm 0.02$ & $0.36 \pm 0.0$ \\
\hline
\end{tabular}




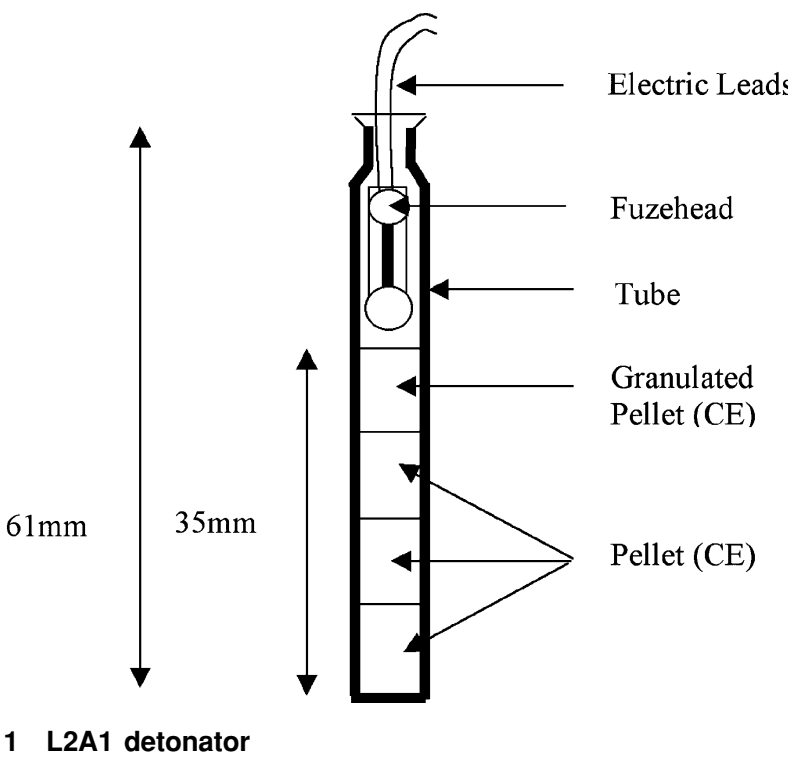

each with a nominal mass of $0 \cdot 25 \mathrm{~g}$. The primary charge which tops the main charge consists of composition ASA with a nominal mass of $0.35 \mathrm{~g}$. The detonator is initiated electrically. $\mathrm{CE}\left(\mathrm{C}_{7} \mathrm{H}_{5} \mathrm{~N}_{5} \mathrm{O}_{8}\right)$ has a detonation velocity of $7570 \mathrm{~m} \mathrm{~s}^{-1}$ for a density of $1710 \mathrm{~kg} \mathrm{~m}^{-3} \cdot{ }^{17}$ The nominal density of tetryl pellets is $1590 \mathrm{~kg} \mathrm{~m}^{-3}$, indicating a probable detonation velocity of slightly less than $7570 \mathrm{~m} \mathrm{~s}^{-1}$.

It can be seen from Fig. 1 that the part of the detonator containing the main filling is $35 \mathrm{~mm}$ in length. This determines the length of the sample cylinders.

Each experiment was conducted inside a bucket (Fig. 2) in order to contain any fragments generated. The cylinder was then placed in the tube of cardboard and the detonator was pushed into the hole in the middle of the cylinder. A second sheet of cardboard was then placed over the top of the cardboard tube and the remainder of the bucket was filled with sand. The lid was then placed on the bucket and was weighted down with a sandbag. The detonator wires trailed from underneath the lid and were connected to an initiating device.

On initial inspection, the bucket remained intact and all fragments were captured within the sand. In each case, the sand was sifted to find all the fragments, and this was verified by first cleaning the fragments of sand and embedded cardboard, and then comparing the mass of the fragments retrieved with the original unexploded mass of each block.

\section{Results}

\section{Visual examination}

The fragments from the 15-42 $\mathrm{mm}$ diameter cylinders can be seen in Fig. 3. Visually, as the cylinder size decreases, the fragments get smaller and, for constant cylinder diameter, as the alloy gets softer, the fragments

Table 4 Charge: metal $(C / M)$ ratios for cylinders

\begin{tabular}{ll}
\hline Diameter, mm & $C / M(M / C)$ \\
\hline 15 & $0.0752(13 \cdot 3)$ \\
20 & $0.0382(26 \cdot 2)$ \\
25 & $0.0231(43 \cdot 3)$ \\
33 & $0.0130(76 \cdot 9)$ \\
42 & $0.0079(126 \cdot 0)$ \\
50 & $0.0055(182 \cdot 0)$ \\
100 & $0.0014(730 \cdot 0)$ \\
\hline
\end{tabular}

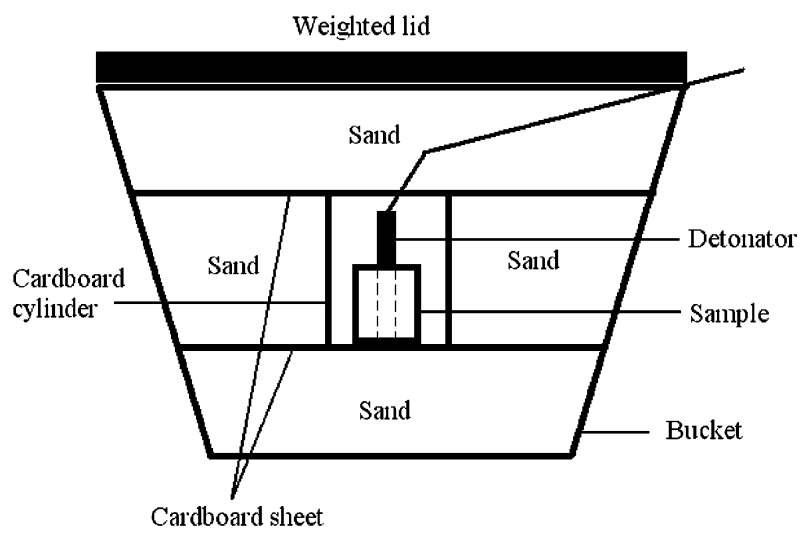

2 Experimental arrangement of cylinder in sand bucket

get larger. At larger diameters (Fig. $3 a-f$ ), the fragments are the length of the original cylinder, while at smaller diameters, the fragments get shorter and more of them become essentially equiaxed in form.

When the larger cylinders were examined, it was seen that the $50 \mathrm{~mm}$ as received sample was on the verge of fragmenting into three fragments with fractures parallel to the cylinder axis as shown in Fig. 4.

There was enlargement of the central hole, initially $6.5 \mathrm{~mm}$ diameter, as seen in Fig. 5. This enlargement (Table 5) was more marked at the bottom of the cylinder than at the top, greater for the softer alloy tempers and somewhat greater for the $50 \mathrm{~mm}$ diameter than for the $100 \mathrm{~mm}$ diameter cylinders.

For the other large cylinders, it was noted that there was some spalling at the base of the cylinder.

\section{Fragment masses}

The numbers and masses of the individual fragments were determined for the cylinders (15-42 $\mathrm{mm}$ diameter) that fragmented. The recovery of fragments was close to $100 \%$, although some of the finer fragments produced in the 15 and $20 \mathrm{~mm}$ cylinders were not recovered as can be seen in Table 6. Figure 6 confirms the conclusions of the visual examination that the fragmentation gets finer with decreasing cylinder diameter (lower $M / C$ ) and coarser as the alloy becomes softer.

The cumulative numbers of fragments heavier than a given mass $M$ are given in Table 7 for the cylinders (15$42 \mathrm{~mm}$ diameter) that fragmented.

Some of the data of Table 7 are presented as a function of cylinder diameter (heat treatment constant) in Fig. 7 and as a function of heat treatment (cylinder diameter constant) in Fig. 8.

\section{Discussion of results}

When the effect of varying the $C / M$ ratio is considered, it is useful to divide the fragmentation into three distinct Table 5 Hole diameter increase in 50 and $100 \mathrm{~mm}$
diameter samples

\begin{tabular}{lll}
\hline Sample & $\begin{array}{l}\text { Top hole diameter, } \\
\mathrm{mm}\end{array}$ & $\begin{array}{l}\text { Bottom hole diameter, } \\
\mathrm{mm}\end{array}$ \\
\hline 100AR & $6 \cdot 66$ & $10 \cdot 52$ \\
100ST & $6 \cdot 53$ & $12 \cdot 16$ \\
1000A & 6.52 & $15 \cdot 66$ \\
50AR & $7 \cdot 78$ & $12 \cdot 34$ \\
50ST & 6.62 & $13 \cdot 6$ \\
50OA & 6.9 & $15 \cdot 7$ \\
\hline
\end{tabular}



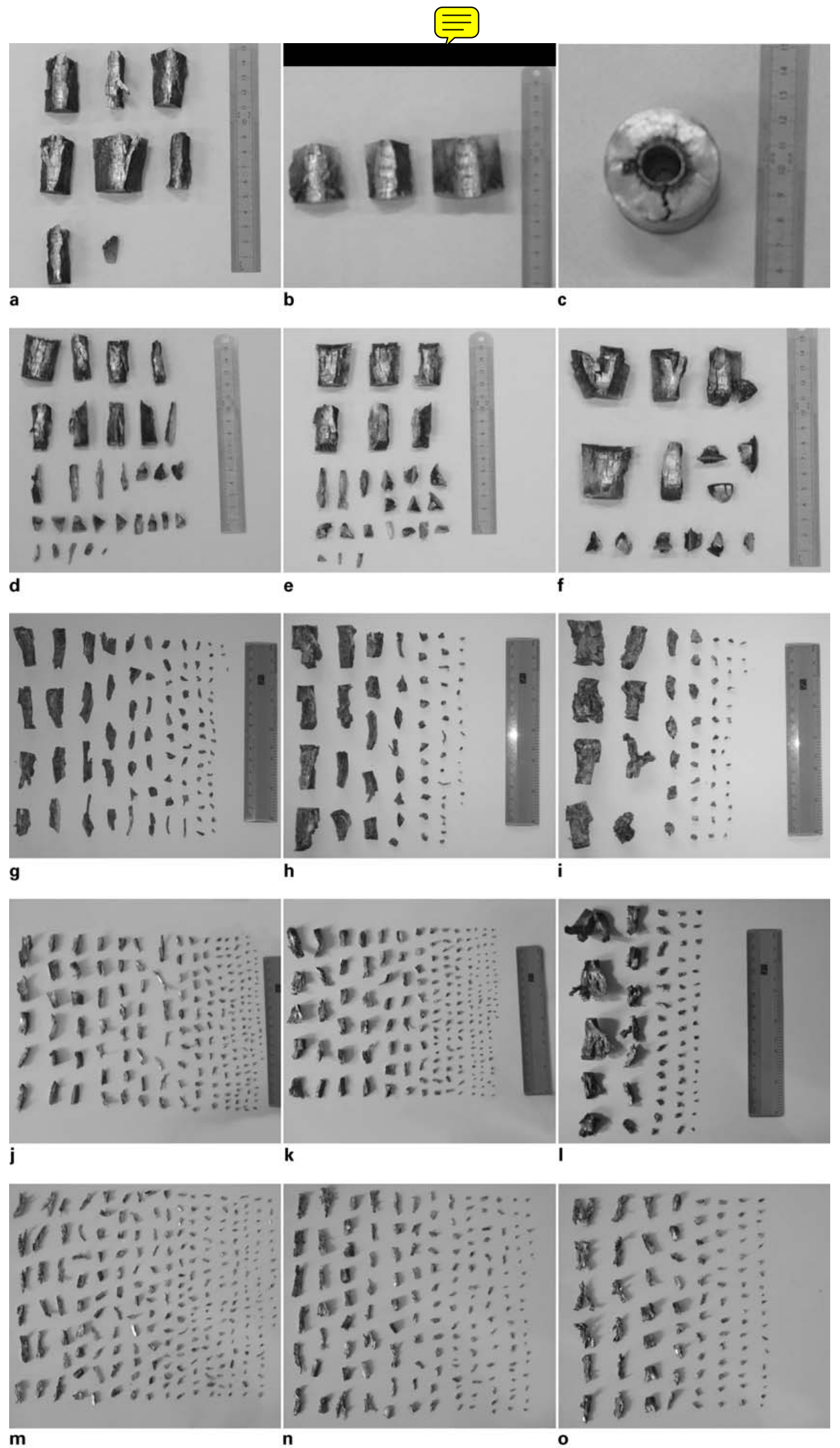

a 42AR; $b$ 42ST; c 42OA; $d$ 33AR; e 33ST; $f$ 33OA; $g$ 25AR; $h$ 25ST; $i$ 25OA; $j$ 20AR; $k$ 20ST; $I$ 200A; $m$ 15AR; $n$ 15ST; o $150 \mathrm{~A}$

3 Visual observation of fragments (15-42 $\mathbf{~ m m}$ diameter cylinders)

regimes. The first at low $C / M$ sees enlargement of the hole, this being larger at the bottom than at the top (Table 5) and greater at higher $C / M$ (Table 5 and Figs. 4 and 5), with no break-up into fragments, except by spalling as the shock wave is reflected at the bottom of the cylinder. In the second $C / M$ regime, fragmentation is in the form of long cylinders (Fig. $3 a-f$ ), while at the highest values of $C / M$, fragmentation is in the form

Materials Science and Technology 2009 VOL 000 NO 000 


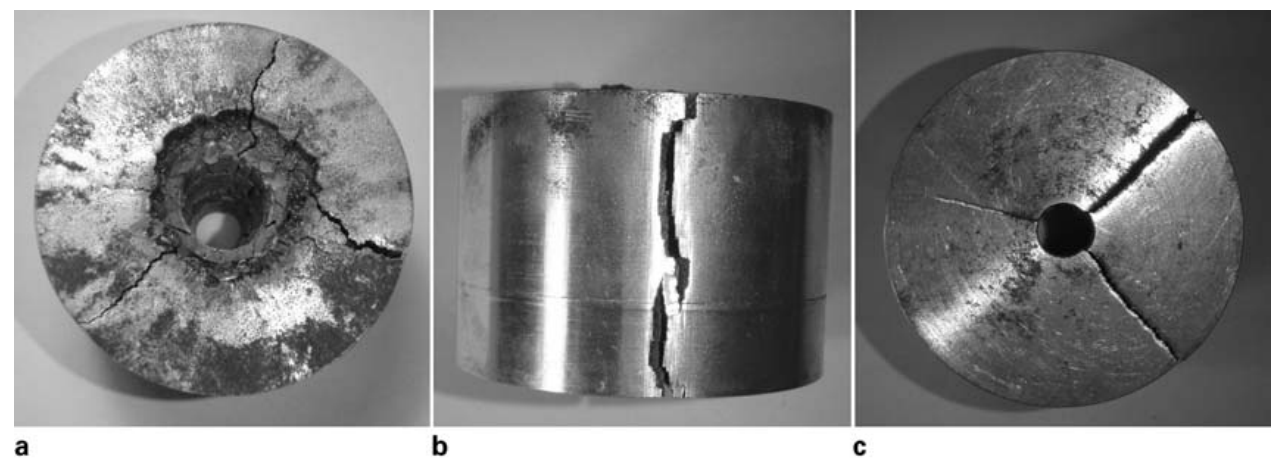

ONLINE

COLOUR

ONLY

a bottom; $b$ side; $c$ top

4 As received sample of $50 \mathrm{~mm}$ diameter showing beginnings of fragment formation: note spalling in a

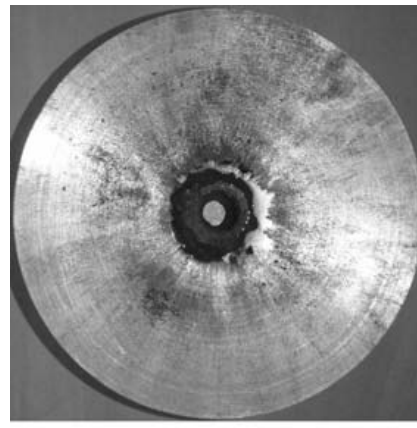

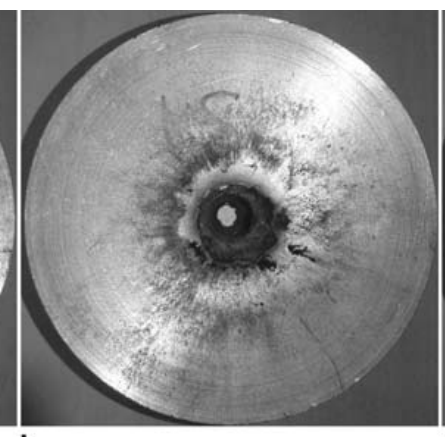

b

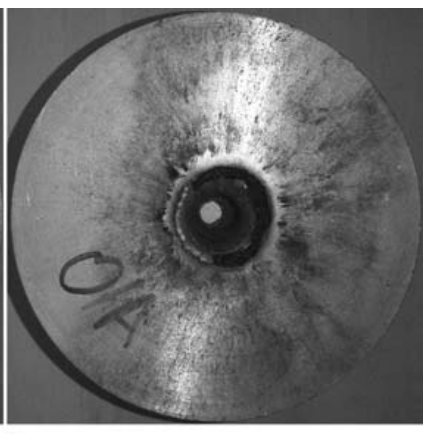

ONLINE

COLOUR

ONLY

$a$ as received; $b$ solution treated; $c$ overaged

5 Samples of $100 \mathrm{~mm}$ diameter showing difference in bottom hole diameter

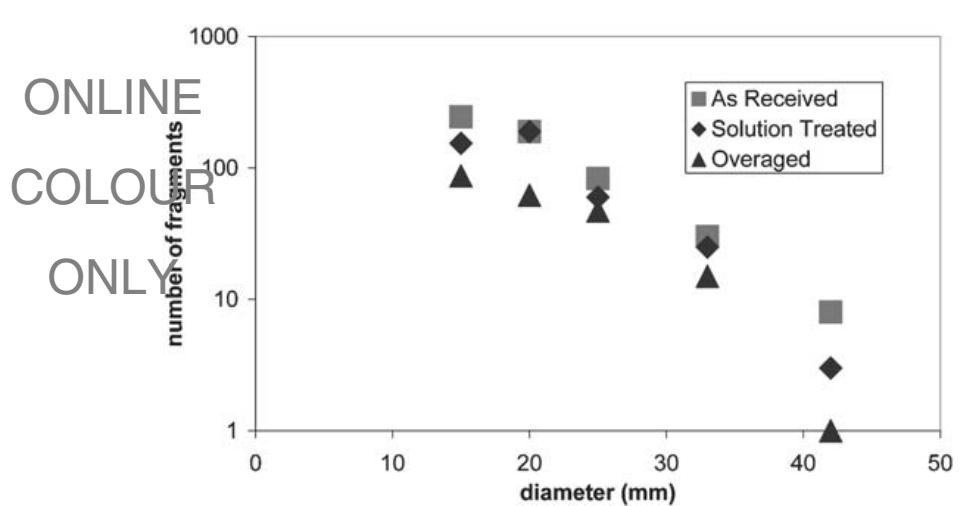

6 Number of fragments as function of heat treatment and cylinder diameter of shards which can be long and thin or essentially equiaxed in shape (Fig. $3 g-o$ ).

Radial expansion that increases as a detonation wave passes down the axis of a cylinder filled with explosive was first noted by Taylor ${ }^{18}$ and the phenomenon has been used in the cylinder expansion test to evaluate the equations of state for explosives. ${ }^{19,20}$ For the lowest values of $\mathrm{Cl} M$, there is deformation without cracking (Fig. 5). At a slightly higher $C / M$, there is cracking present in the as received sample (Fig. 4) showing that the hoop strain for fracture is exceeded at the bottom of the cylinder. At $C / M=0 \cdot 0079$ and $0 \cdot 013$, break-up is divided into a small number of fragments with longitudinal fractures along the whole length of the cylinder. This pattern of fragmentation is similar to that reported for large thick walled cylinders of annealed AISI 1020

Table 6 Numbers and masses of fragments retrieved (15-42 mm diameter samples)

\begin{tabular}{lcccc}
\hline Sample & No. of Fragments & Original mass, & Mass of fragments, g & Recovered, \% \\
\hline 42AR & 8 & $132 \cdot 5$ & $130 \cdot 6$ & $98 \cdot 6$ \\
42ST & 3 & $132 \cdot 5$ & $132 \cdot 3$ & $99 \cdot 8$ \\
42OA & 1 & $132 \cdot 6$ & $132 \cdot 6$ & 100 \\
33AR & 30 & $80 \cdot 8$ & $79 \cdot 5$ & $98 \cdot 5$ \\
33ST & 25 & $80 \cdot 7$ & $80 \cdot 1$ & $99 \cdot 3$ \\
33OA & 15 & $80 \cdot 7$ & $80 \cdot 4$ & $99 \cdot 7$ \\
25AR & 83 & $45 \cdot 4$ & $44 \cdot 0$ & $96 \cdot 9$ \\
25ST & 60 & $45 \cdot 6$ & $45 \cdot 0$ & $98 \cdot 6$ \\
25OA & 47 & $45 \cdot 5$ & $45 \cdot 5$ & 100 \\
20AR & 189 & $27 \cdot 5$ & $25 \cdot 9$ & $94 \cdot 2$ \\
20ST & 189 & $27 \cdot 6$ & $27 \cdot 6$ & 100 \\
200A & 62 & $27 \cdot 4$ & $27 \cdot 0$ & $98 \cdot 5$ \\
15AR & 245 & $14 \cdot 0$ & $12 \cdot 9$ & $92 \cdot 1$ \\
15ST & 154 & $13 \cdot 9$ & $12 \cdot 9$ & $92 \cdot 8$ \\
15OA & 87 & $14 \cdot 0$ & $13 \cdot 9$ & $99 \cdot 3$ \\
\hline
\end{tabular}




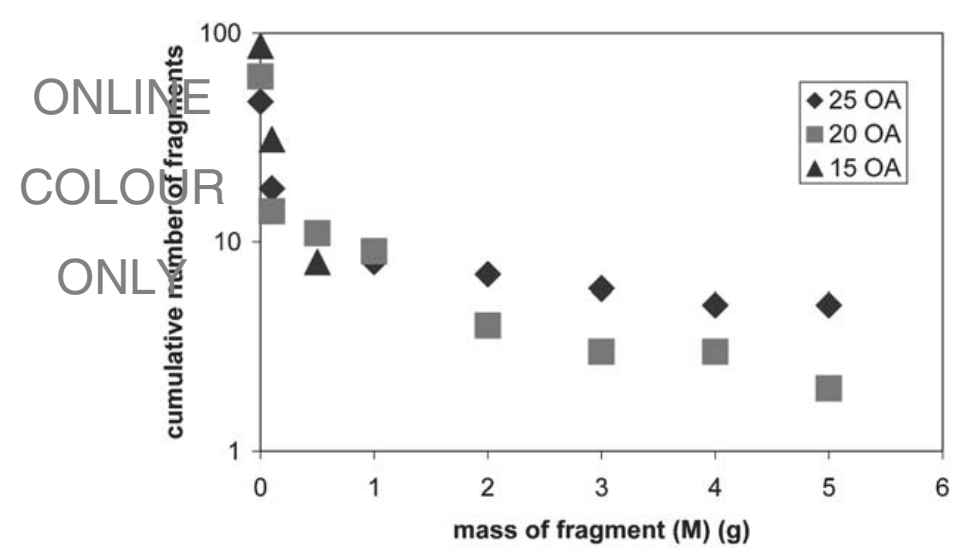

7 Cumulative numbers of fragments for various cylinder diameters

steel by Pearson and Rinehart. ${ }^{11}$ At higher values of $C / M$, the fragmentation pattern changes again with hoop strains being exceeded soon after initiation of the detonator. Since the velocity of crack propagation is less than the Rayleigh wave velocity, ${ }^{21,22}$ this is substantially slower than the detonation velocity of the explosive in the detonator, and therefore, cracking and fragmentation will start along the length of the cylinder as the detonation wave passes along the cylinder length. The fragments are likely to be of different shapes, as is seen in Fig. $3 g-O$ and confirmed, following Mock and Holt, ${ }^{8}$ by the bilinear cumulative fragment distributions of Figs. 7 and 8. The number of fragments produced increased as the $C / M$ ratio increased, as shown in Fig. 6 for all heat treatment conditions and for both the patterns of fragmentation seen in Fig. 3. For the thin walled cylinders, this would be as predicted by equations (1) and (2), since the velocity at break-up in equation (1) will increase with $C / M$ as will the strain rate in equation (2). In the case of thick walled cylinders, the particle velocity increases with $C / M$, thus increasing the number of fragments when the external diameter changes from 42 to $33 \mathrm{~mm}$.

Fragmentation is known to be affected by the mechanical properties of the metal. Equations (1) and (2) for the fragmentation of thin walled cylinders predict that the more ductile overaged samples should produce fewer fragments at a given $C / M$ ratio than would the stronger as received (peak aged) samples. This is shown

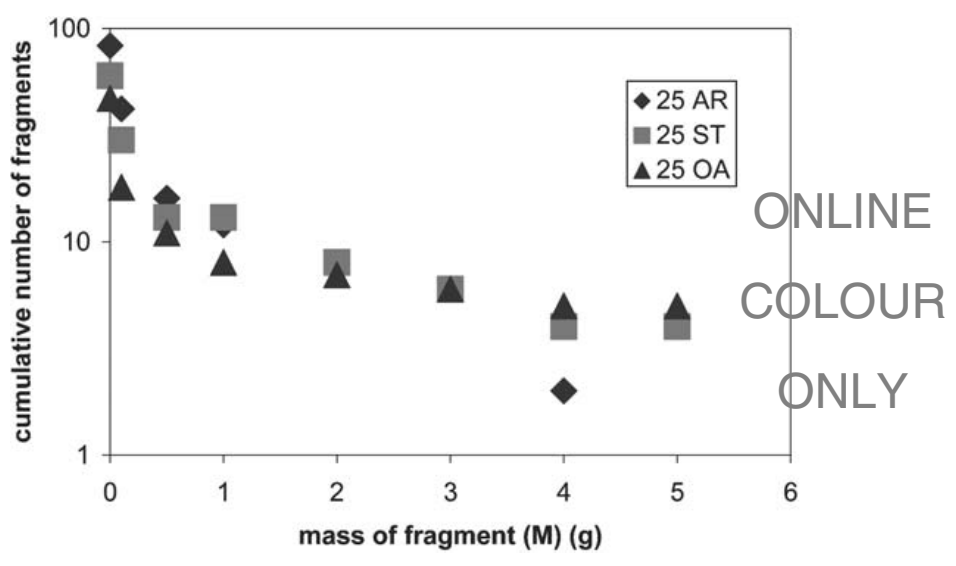

8 Cumulative numbers of fragments as function of alloy heat treatment

in Table 6. Results for the solution treated samples should produce an intermediate number of fragments which they do. It is of interest to look at equations (1) and (2) more quantitatively. The assumption ${ }^{5}$ made in equation (1) is that the cylinder is in the form of a ring bomb, which has a uniform length in the $z$ direction, and that all fractures producing fragments are in the radial direction with fragments containing both inner and outer cylinder walls (type 1 in the taxonomy of Mock and $\mathrm{Holt}^{8}$ ). Since the fragments created are of varying morphologies, quantitative analysis would not be particularly helpful. In contrast, it is possible to make came estimate of the effect of changing mechanical $\equiv$ perties on equation (2). If it is assumed that the mass of a fragment is proportional to $S^{3}$, then equation (2) can be rewritten as

$$
S=a K_{\mathrm{f}}
$$

Using estimates of plane strain fracture toughness of 2 $25 \mathrm{MPa} \mathrm{m}^{1 / 2}$ for $7075-\mathrm{T}^{23}$ and $50 \mathrm{MPa} \mathrm{m}^{1 / 2}$ for the overaged sample, ${ }^{24}$ assuming a yield strength of $275 \mathrm{MPa}$ would indicate that the average fragment mass should be increased by a factor of 2 when going from the strongest AR to the weakest OA condition. Results from Table 6 give factors of $>2 \cdot 8,>3 \cdot 0$ and $>1 \cdot 8$ for diameters of 15,20 and $25 \mathrm{~mm}$, considering all fragments. The 'greater than' sign is put since the mass collected was not $100 \%$. This suggests that an estimate of the effect of a change in heat treatment on the average

Table 7 Cumulative numbers of fragments with mass greater than $M$ (15-42 $\mathrm{mm}$ diameter samples)

\begin{tabular}{|c|c|c|c|c|c|c|c|c|c|c|c|c|}
\hline \multirow[b]{2}{*}{$M \rightarrow$} & \multicolumn{12}{|c|}{ No. of fragments of mass greater than $M, \mathrm{~g}$} \\
\hline & 0 & 0.005 & 0.01 & 0.05 & $0 \cdot 1$ & 0.5 & 1 & 2 & 3 & 4 & 5 & 10 \\
\hline 42AR & 8 & 8 & 8 & 8 & 8 & 8 & 7 & 7 & 7 & 7 & 7 & 7 \\
\hline 42ST & 3 & 3 & 3 & 3 & 3 & 3 & 3 & 3 & 3 & 3 & 3 & 3 \\
\hline $420 \mathrm{~A}$ & 1 & 1 & 1 & 1 & 1 & 1 & 1 & 1 & 1 & 1 & 1 & 1 \\
\hline 33AR & 30 & 30 & 30 & 29 & 29 & 21 & 10 & 8 & 8 & 8 & 8 & 1 \\
\hline 33ST & 25 & 25 & 25 & 25 & 23 & 17 & 10 & 6 & 6 & 6 & 6 & 4 \\
\hline $330 \mathrm{~A}$ & 15 & 15 & 15 & 14 & 14 & 13 & 9 & 5 & 5 & 5 & 5 & 4 \\
\hline 25AR & 83 & 83 & 79 & 52 & 42 & 16 & 12 & 8 & 6 & 2 & $\ldots$ & $\ldots$ \\
\hline 25ST & 60 & 58 & 53 & 34 & 30 & 13 & 13 & 8 & 6 & 4 & 4 & $\ldots$ \\
\hline $250 A$ & 47 & 46 & 38 & 21 & 18 & 11 & 8 & 7 & 6 & 5 & 5 & $\ldots$ \\
\hline 20AR & 189 & 174 & 148 & 69 & 54 & 19 & 6 & $\ldots$ & $\ldots$ & $\ldots$ & $\ldots$ & $\ldots$ \\
\hline 20ST & 189 & 150 & 114 & 53 & 44 & 20 & 6 & 2 & $\ldots$ & $\ldots$ & $\ldots$ & $\ldots$ \\
\hline $200 A$ & 62 & 61 & 55 & 23 & 14 & 11 & 9 & 4 & 3 & 3 & 2 & $\ldots$ \\
\hline 15AR & 245 & 219 & 177 & 65 & 43 & $\ldots$ & $\ldots$ & $\ldots$ & $\ldots$ & $\ldots$ & $\ldots$ & $\ldots$ \\
\hline 15ST & 154 & 148 & 122 & 54 & 38 & 2 & $\ldots$ & $\ldots$ & $\ldots$ & $\ldots$ & $\ldots$ & $\ldots$ \\
\hline $150 \mathrm{~A}$ & 87 & 84 & 80 & 38 & 31 & 8 & $\ldots$ & $\ldots$ & $\ldots$ & $\ldots$ & $\ldots$ & $\ldots$ \\
\hline
\end{tabular}


fragment size can be made using equation (2). It is still important to accept that there will be a large variation in fragment sizes, as seen in Table 7, and that fragment distributions will be important in determining the effectiveness of any fragmentation. Considering the fracture of thick walled cylinders (33 and $42 \mathrm{~mm}$ outside diameters), the number of fragments produced is the greatest for the cylinders in the as received condition and the smallest for those that had been overaged. Indeed, in the as received condition, the onset of fragmentation can be seen for the $50 \mathrm{~mm}$ diameter cylinders (Fig. 4), something not seen for the $50 \mathrm{~mm}$ diameter cylinders in the other heat treatment conditions. Since the critical impact velocity tends to decrease with increasing alloy hardness, ${ }^{25}$ an increase in number of fragments (Fig. 6) seen in the as received condition would be expected and also with the solution treated alloy producing more fragments than the overaged. For the thin walled cylinders, the fragmentation in the as received condition is finer both measured by the total number of fragments and the mass of the largest fragment (Table 7 and Fig. 8). Again the coarsest fragmentation is that seen in the overaged samples, even though the pattern of fragmentation is different from that seen in the thick cylinders of 33 and $42 \mathrm{~mm}$ diameters.

Taylor $^{18}$ has estimated the shape of a cylinder detonated at one end and showed that the diameter of the central hole increases with distance from the initiation point and will be greater for higher values of $C / M$. The results of Table 5 confirm this for this work with hole diameters greater for the $50 \mathrm{~mm}$ diameter cylinders and the bottom hole diameter greater than the top hole diameter. Hole enlargement at constant $\mathrm{ClM}$ is the greatest for the softest overaged material and the least for the strongest peak aged material. This would be as expected as the strain to fracture will be the greatest for the alloy in its softest condition.

\section{Conclusions}

1. The small scale testing, by the use of varying $C / M$ ratios, can produce patterns of fragmentation similar to that seen in the large scale testing of cylinders packed with military explosives.

2. The fineness of fragmentation increases when the aluminium alloy is in the highest strength (peak aged) condition and decreases when the alloy is in its lowest strength (overaged) condition. This applies for both thin and thick walled cylinders.

3. There is an increase in the fineness of fragmentation seen when the $C / M$ ratios of both thick and thin walled cylinders are increased.

4. For thin walled cylinders, there are varying shapes of fragment, indicating that fracture occurs by more than one mechanism.
5. The enlargement of the central hole in nonfragmenting specimens is the greatest for the highest $C / M$ cylinders and for constant $C / M$, the highest for the softest overaged specimens.

\section{Acknowledgement}

The authors wish to thank their Cranfield colleagues A. Mustey (mechanical testing) and J. Clements (range support) for their help in completing this project.

\section{References}

1. R. Gurney: 'The initial velocities of fragments in bombs, shells and grenades', Report 405, Ballistic Research Laboratory, Aberdeen, MD, USA, 1943 (cited in M. A. Meyers: 'Dynamic behavior of materials', 234-242; 1994, New York, John Wiley \& Sons).

2. E. V. Esquivel and L. F. Murr: Mater. Sci. Technol., 2006, 22, (4), 438-452.

3. M. R. Edwards: Mater. Sci. Technol., 2006, 22, (4), 453-462.

4. B. A. Remington, P. Allen, E. M. Bringa, J. Hawreliak, D. Ho, K. T. Lorenz, H. Lorenzana, J. M. McNaney, M. A. Meyers, S. W. Pollaine, K. Rosolankova, B. Sadik, M. S. Schneider, D. Swift, J. Wark and B. Yaakobi: Mater. Sci. Technol., 2006, 22, (4), 474 488.

5. N. F. Mott: Proc. R. Soc. Lond. A, 1947, 189A, 300-308.

6. D. E. Grady: J. Appl. Phys., 1982, 53, 322-325.

7. D. E. Grady and M. L. Olson: Int. J. Impact Eng., 2003, 29, 293306.

8. W. Mock and W. H. Holt: J. Appl. Phys., 1983, 54, 2344-2351.

9. D. E. Grady and M. E. Kipp: Int. J. Impact Eng., 1997, 20, 293 308

10. D. E. Grady and M. E. Kipp: J. Appl. Phys., 1985, 58, 8025-8030. J. Pearson and J. S. Rinehart: J. Appl. Phys., 1952, 23, 434-441.

J. S. Rinehart and J. Pearson: 'Behavior of materials under impulsive loads', 136-137; 1954, New York, Dover Press.

13. D. P. Lidstone: 'Cartridge case deformation test', RARDE Memorandum 15/69, PARDE, Surrey, UK, 1969.

14. A. Lowe and R. Hiley: J. Energ. Mater., 1998, 16, 289-307.

15. J. C. Oxley, J. L. Smith and E. Resende: J Forensic Sci., 2001, 46, (5), 1070-1075.

16. B. E. Walsh: 'Comparison of natural fragmentation scaling behavior of HF-1 and AISI 1045 steel cylinders using normalized Payman parameters', Report MRL-R-941, DSTO Materials Research Laboratories, Ascot Vale, Vic., Australia, 1984.

7. R. Meyer, J. Köhler and A. Homburg: 'Explosives', 6th edn, 311; 2007, Weinheim, Wiley-VCH Verlag.

18. G. I. Taylor: 'The scientific papers of Sir Geoffrey Ingram Taylor', Vol. III, 277-286; 1963, Cambridge, Cambridge University Press.

19. C.-Y. Chen, J.-H. Shiuan and I.-F. Lan: Propell. Explos. Pyrotech., 1994, 19, (1), 9-14.

20. R. Catanach, L. Hill, H. Harry, E. Aragon and D. Murk: 'Cylinder test specification', Report LA-13643-MS, Los Alamos National Laboratory, Los Alamos, NM, USA, 1999.

21. M. A. Meyers: 'Dynamic behavior of materials', 496-522; 1994, New York, John Wiley \& Sons.

22. A. T. Zehnder and A. J. Rosakis: Int. J. Fract., 1990, 43, 271285.

23. Available at: http://www.matweb.com (accessed 29 July 2009).

24. I. J. Polmear: 'Light alloys', 4th edn, 135; 2006, Oxford, Butterworth-Heinemann (cited in R. Develay: Met. Mater., 1972, 6, 404 410).

25. D. S. Clark and D. S. Wood: Trans. ASM, 1950, 42, 45-52. 


\section{Authors Queries}

Journal: Materials Science and Technology

Paper: 9136

Title: Effect of heat treatment on small scale fragmentation of aluminium alloy

Dear Author

During the preparation of your manuscript for publication, the questions listed below have arisen. Please attend to these matters and return this form with your proof. Many thanks for your assistance

\begin{tabular}{|l|l|l|}
\hline $\begin{array}{l}\text { Query } \\
\text { Reference }\end{array}$ & Query & Remarks \\
\hline 1 & $\begin{array}{l}\text { Author: Please confirm the run- } \\
\text { ning head. }\end{array}$ & \\
\hline 2 & $\begin{array}{l}\text { Author: Please confirm the } \\
\text { change of equation. }\end{array}$ & \\
\hline
\end{tabular}

